\title{
Diagnosing Uncertainty, Producing Neonatal Abstinence Syndrome (NAS)
}

\section{Authors}

Amy Chandler, School of Health in Social Science, University of Edinburgh, a.chandler@ed.ac.uk (corresponding author)

Anne Whittaker, NMAHP Research Unit, University of Stirling

Sarah Cunningham-Burley, Usher Institute, University of Edinburgh

Lawrie Elliott, Department of Nursing and Community Health, Glasgow Caledonian University

Paula Midgley, University of Edinburgh/NHS Lothian

Sarah Cooper, NHS Lothian

\section{Abstract (199 words)}

The use of alcohol and other drugs during pregnancy is understood to be an important public health problem. One way in which this problem is expressed and responded to is via the identification and treatment of Neonatal Abstinence Syndrome (NAS). In this paper, we demonstrate how the processes of anticipating, identifying and responding to NAS are characterised by significant uncertainty among parents and health and social care practitioners. We draw on interviews with 16 parents who had recently had a baby at risk of NAS, and multi-disciplinary focus groups with 27 health and social care professionals, held in Scotland, UK. NAS, and drug-use in pregnancy, is a fraught and complex arena. Parents in the UK who use opioids risk losing custody of children, and must navigate a high degree of surveillance, governance and marginalisation. We suggest that considering NAS as a social diagnosis, further informed by Mol's political ontology of 'multiple' bodies/diseases, may help to produce clinical and social responses to uncertainty which avoid, rather than promote, further marginalisation of parents who use drugs. One such response is to develop a culture of relationship-based care which empowers both service providers and service users to challenge existing practice and decision making.

Word count: 7883

\section{Introduction}

Neonatal Abstinence Syndrome (NAS) refers to a 'constellation' of symptoms associated with drug withdrawal in babies born to mothers who use certain types of drugs during pregnancy (Jansson \& 
Special Issue: Uncertainty

Velez, 2012). It was initially popularised in a 1975 paper by Finnegan and colleagues (Marcellus, 2007), and is regularly said to be an increasing public health problem, particularly given rising availability of prescription opioids in the United States and Canada (Pryor et al., 2017). NAS is primarily associated with opioid use during pregnancy, but has been described in babies born to mothers who use a range of psychoactive drugs (including cannabis, amphetamines and benzodiazepines), though reporting is inconsistent (Hudak et al., 2012). Indeed, there is a dearth of understanding about the impacts of poly-drug use on NAS, despite poly-drug use being the norm among certain groups of women (e.g. those who are prescribed methadone) (McQueen et al., 2015).

NAS can be understood as a contested and controversial diagnostic label. The identification of NAS can lead to legal repercussions for the mother - including prosecutions for 'child abuse', or losing custody of children (Boyd, 1999; Gregory, 2010). Although NAS is said to occur in relation to an assortment of substances, it remains closely associated with those understood as 'problematic' or illicit. Worldwide, there are variable and conflicting responses to substance use during pregnancy ranging from supportive interventions aimed at enhancing parenting capacity and child welfare, along with maintaining family bonds (Knopf, 2016; Morris et al., 2012; Sword et al., 2009); to coercive, punitive responses where drug-using pregnant women are framed as inherently unsuitable and dangerous, 'notification' to social services is mandatory, and legal measures are used to remove children, sometimes shortly after birth (Boyd, 1999; Flavin \& Paltrow, 2010; Gregory, 2010; Kenny et al., 2015; Knight, 2015).

The study of NAS, and in particular what it means to various actors involved in responding to and constructing the diagnosis, is essential in unpicking an already complex and contested area: drug use during pregnancy and early parenthood (Chandler et al., 2013; Kilty \& Dej, 2012; Radcliffe, 2009). While there may be severe repercussions if NAS is identified, there is also a great deal of disagreement and variability in clinical management of such babies. NAS is identified in different ways, using different measures and score charts (Hudak et al., 2012; Wolff \& Perez-Montejano, 2014). Similarly, treatments for NAS differ - in some contexts, pharmacological treatment and care in isolated neonatal units are standard practice (Hudak et al., 2012), in others, non-invasive approaches which emphasise mother-child bonding in non-clinical settings are emphasised (Jansson \& Velez, 2012; Knopf, 2016).

Despite these variations, research on local meanings and practices relating specifically to NAS are limited. Wider studies addressing drug-use in pregnancy or parenthood have identified diverse practices relating to NAS, highlighting potentially problematic features (Boyd, 1999; Klee et al., 2002; Knight, 2015). However, few qualitative studies focusing specifically on NAS exist (Atwood et al., 
Special Issue: Uncertainty

2016; Benoit et al., 2014; Cleveland \& Bonugli, 2014). While offering some insight into the complex challenges faced by parents and practitioners alike, existing studies tend to be clinically driven, relatively descriptive and do not engage deeply with the social, cultural or material contexts in which care for babies at risk of NAS takes place. Sociological attention regarding these issues is particularly sparse, though with regard to drug use in general, sociological perspectives have been widely and successfully applied (e.g. Fraser et al., 2014; Fraser \& valentine, 2008).

Our study aimed to explore understandings of NAS among parents whose babies were at risk of NAS (all mothers used opioids during pregnancy), and among health and social care practitioners experienced in working with such parents, or their babies (AUTHOR 2019). The study examined local understandings of NAS in two neighbouring geographical areas of Scotland, UK. To make sense of the multiple ways in which NAS was constructed in these accounts, and the diverse resources drawn upon to produce NAS, we draw on scholarship addressing the sociology of diagnosis, and specifically Brown's concept of social diagnosis (2011). Uncertainty was a theme throughout the findings, and we reflect on the importance of working with such uncertainty for practitioners, and managing this for parents, particularly in the context of child protection policies and practices which mark parental, and especially maternal, substance use as fundamentally problematic, and drug-exposed infants as inherently 'at risk'.

\section{Social Diagnosis and NAS}

While sociology has long been concerned with the social construction and contexts in which diagnosis plays out (Blaxter, 1978; Brown, 1990), the 'sociology of diagnosis' has received special attention in recent years (Jutel, 2009; Jutel \& Nettleton, 2011). In this paper, we draw on the work of Brown and colleagues, and their concept of social diagnosis (2011).

The concept of social diagnosis explicitly expands the scope of sociological analysis of diagnoses to incorporate wider structural and cultural contexts, engaging with the multiple actors and resources who may be engaged in diagnostic work - including sociologists (Brown et al., 2011; Shriver \& Bodenhamer, 2018). This approach facilitates an examination of pathogenic structural and cultural factors which might promote or inhibit particular forms of disease, or facilitate the identification or diagnosis of a given disease (2011 p. 939). This is a potentially productive way of considering NAS, and more broadly 'problematic' drug use: neither is evenly spread throughout populations, but are more likely to be concentrated in the bodies of those who are marginalised and poor (Knight, 2015; Powis et al., 2000). Despite the clear relevance of social, economic and political factors, discourse about both NAS and problem drug often focuses on psychological or biological pathologies, with addiction more often framed as an individual affliction (Vrecko, 2010). Where 'the social' is present, 
Special Issue: Uncertainty

this tends to be limited to individual life histories marked by trauma, abuse and loss. In relation to drug-use and parenting (including discussions of NAS), such factors are associated with attendant problems with 'attachment' and quality of caregiving, which are then related to likely problems in future parenting capacity and child welfare (ACMD, 2003). The social diagnosis of NAS supports a sociological gaze which can take into account structural and symbolic violence as contributing to the material and social production of this particular condition and to understanding how and why it is identified in particular bodies (and, perhaps, not others). Such a gaze may have the potential effect of diverting attention away from individual (pathological) maternal bodies, and provide a basis for an active sociological intervention into ongoing debates about the governance of drug-use in the context of parenting.

The concept of social diagnosis (at least as expressed in Brown et al's 2011 paper) is more limited in how far it engages with intimate, embodied, material practices of diagnosis. There is a danger when considering broad structural and cultural issues, of losing some of the significant interpersonal, intercorporeal and material contexts which also 'make up' particular diagnostic processes. This distance from intimate settings may also contribute to a relative lack of engagement with resistance to diagnostic processes (Jenkins \& Short, 2017). We propose an approach which bridges this gap: connecting the concept of social diagnosis to Mol's work on the production of the 'body multiple' via different forms of clinical practice (Mol, 2002). This approach resonates with Jenkins and Short's recent modification of social diagnosis, to include greater attention to processes of resistance to diagnosis. Like Mol's approach to the 'body multiple', Jenkins and Short's argument similarly underlines multiplicity in how diagnosis takes place. Drawing on debates about the identification and (potential) 'treatment' of intersex people, they note that contested or uncertain diagnoses may be resisted by some, at the same time that others seek to confirm or institutionalise a particular diagnosis and attendant treatment response.

However, Jenkins and Short's analysis of diagnostic resistance in the case of intersex draws on a literature review, and thus also maintains a distance from the interpersonal, intimate processes by which diagnoses may be resisted, contested or confirmed. In contrast, Mol's ethnographic approach engages directly with the rich and multi-layered manner in which diagnosis proceeds in different clinical settings, addressing diversities among material resources; healthcare systems and organisations, clinical specialities, with their attendant priorities and policy drivers; and diverse 'lay' actors, who also draw on available cultural and social resources through which they can make sense of and respond to a diagnosis. Importantly, Mol's work allows analysts to view a 'diagnosis' as multiple. In the case of NAS, as we will demonstrate, this is particularly apposite, since the 'NAS' that 
Special Issue: Uncertainty

is talked of (and feared) by parents, is not necessarily the same as the 'NAS' that is produced by particular score charts, or the 'NAS' that is discussed by social work practitioners as a risk factor endangering a child's future health. Similarly the 'NAS' that midwives working on a standard postnatal ward describe might be different from the 'NAS' that is responded to in a specialist neonatal unit (NNU). The coexistence of multiple enactments of NAS contributes to - but does not constitute - the uncertainty that we identify across narratives of different social actors involved in caring for babies who might be affected by NAS.

\section{Methods}

This paper is based on a qualitative study of the accounts of 16 parents and 27 practitioners regarding experiences of preparing for and caring for babies at risk of developing NAS. The research was carried out by a multi-disciplinary team of social scientists and clinicians, including an obstetrician, neonatologist and addictions nurse. The aims of the research were to explore understandings and responses to NAS among parents and practitioners. We conducted individual interviews with parents of babies who were at risk of NAS, alongside multi-disciplinary focus groups with health and social care practitioners who were involved in the care of families affected by NAS.

Parents were recruited during pregnancy, and interviews arranged between 1 month and 6 months after babies were born. Recruitment was supported by healthcare practitioners, who approached opioid-dependent women during pregnancy (e.g. women known to services as opioid dependent, in most instances receiving a prescribed opioid-substitute such as methadone). Most participants were informed of the study by their midwives. If women expressed initial interest, they were provided with a participant information sheet, and advised that a researcher would call to discuss further. A phone call was held with interested women, where the research was discussed and explained in more detail. At this point, the researcher raised the possibility of also interviewing the father (social or biological) of the baby. Given high rates of domestic violence experienced among drug-dependent women (Radcliffe et al., 2016), we felt women should hold as much power as possible as to whether their partners were involved in or aware of the research. For women who were still interested, an agreement was made to make further phone contact after the due date, to check in and - if still desired-arrange a time and place for the interview. If women suggested their partner could be contacted, they were called and the research discussed to gauge whether they were interested in taking part in a separate interview.

Interviews addressed parents' history of drug use, contextual features of their lives, experiences during and after pregnancy, with a particular focus on NAS, and interactions with health and social 
Special Issue: Uncertainty

care providers. Interviews with couples were held separately. In most cases, interviews were held in participants' homes.

Alongside the interviews, four interdisciplinary focus groups were conducted with 27 health and social care practitioners who worked with parents who use drugs, or babies at risk of NAS.

Participants were recruited through researchers' existing and well-established links with

practitioners. Focus groups addressed participants' experiences of working with parents on the issue of NAS; recognising and supporting babies experiencing NAS. Professional staff were recruited because of their experience in working with opioid-dependent parents, and included social workers, early years workers, midwives, neonatal nurses, General Practitioners, consultant psychiatrists, obstetricians and neonatologists, addictions nurses, and health visitors (child health nurses). Focus group participants were recruited through the multidisciplinary research team's substantial networks across health and social care services in the areas studied, with potential participants sent email invitations to gauge initial interest. The focus groups were facilitated by two researchers, so that notes could be taken about interactions, group dynamics and non-verbal communication between group members.

The study was held in two neighbouring areas of Scotland, UK. Area 1 includes a large city, Area 2 a large town. Each area incorporates urban and semi-rural populations. The majority of parents interviewed lived in Area 1, with six in Area 2 (three couples). Similarly, the majority of professional participants worked in Area 1, with the exception of four participants, including a neonatal nurse, who worked in Area 2. Maternity care and drug services are organised slightly differently in each area. In particular, urban areas within Area 1 are served by a specialist, multidisciplinary team which cares for pregnant women who are drug or alcohol dependent, and whose substance use is judged to be particularly 'risky' (to themselves, and their future child).

Interviews and focus groups were digitally audio-recorded, transcribed, anonymised and uploaded to NVIVO 10 (QSR International Pty Ltd., 2012). The data were analysed using inductive coding, drawing on narrative and discursive approaches (Hennink et al., 2011; Riessman, 2008). We were interested in how participants talked about - and constructed - NAS in their accounts. All interview and focus group transcripts were initially subject to broad, inductive content coding by Chandler and Whittaker. Following this, particular broad codes of interest were examined in more detail, subject to multiple re-readings, with further sub-codes developed. In this paper, we address a number of related codes that might be characterised as addressing 'NAS talk'. This included discussion about the causes, identification and treatment of NAS. 
Special Issue: Uncertainty

The research was reviewed and approved by the local NHS Research Ethics Committee. All participants provided informed, written consent before data collection; data were fully anonymised and confidentiality maintained. Interviewees received a $£ 20$ gift voucher as a token of thanks for their time and commitment to the study. Focus group participants were provided with lunch.

\section{Findings}

\section{Sample}

16 parents took part in interviews: 7 fathers, and 9 mothers, which included 7 heterosexual couples who were interviewed separately (see Table 1). Parents were aged between 23 and 47, all but two fathers reported current use of opioids, or opioid substitution therapy (methadone, buprenorphine, dihydrocodeine). The majority of the sample reported poly-drug use (including anti-depressant prescriptions, benzodiazepines - prescribed and non-prescribed, cannabis, tobacco and alcohol). Participants talked of living with a range of challenging circumstances. All but one of the participants were unemployed: worries about money were ubiquitous. All were living in social housing (government subsidised), or described unstable housing, including recent periods of homelessness. Twelve participants described previous involvement in the criminal justice system, with nine indicating they had spent time in prison. The majority of participants described limited social support, being estranged from family members and having severed contact with previous 'acquaintances' who continued to use drugs.

[Add Table 1 here]

All of those who took part in the study had custody of their babies at the time of interview. However, six mothers, and five fathers had older children with whom they had limited or no contact. For mothers, this was because children were in the care of others (e.g. they had lost custody); for all but one of the fathers this followed a previous relationship breakdown and estrangement or antagonistic relationships with ex-partners. Four mothers had custody of at least some of their older children.

All participants who described themselves as drug dependent were engaged in drug treatment. Mothers were enrolled on opioid substitution programmes, most were prescribed methadone, with one mother indicating she transferred from methadone to buprenorphine (Subutex) during her pregnancy, and another transferring from methadone to dihydrocodeine. Nine participants described mental health problems, primarily anxiety and depression. Two couples were managed by the multidisciplinary, specialist substance use in pregnancy care team operating in the urban section of Area 1. All other participants received standard maternity care alongside their drug treatment 
Special Issue: Uncertainty

programmes. Drug treatment in Scotland can be provided via primary care or by specialist NHS addictions teams.

Health and social care practitioners who took part in the focus groups were all experienced professionals, who had spent at least five years in their current role. Table 2 provides an overview of focus group participants. Three of the four focus groups included staff from the specialist substance use in pregnancy team.

[Add Table 2 here]

Anticipating the diagnosis, negotiating uncertain causality

The diagnosis or identification of NAS is anticipated during pregnancy, a time where discussions between parents and healthcare providers may begin to address the possibility of NAS, and any steps which could be taken to ameliorate risk of NAS. Indeed, a key concern across both interviews and focus groups was the question of whether or not NAS could be prevented and - relatedly - what 'caused' NAS. Interviewees and focus group participants all readily acknowledged the 'unpredictability' of NAS. This was a common narrative, often supported by anecdotal evidence, which was employed to emphasise that the symptoms of NAS did not correlate neatly with the amount or type of drugs used by women during pregnancy.

"...you could have a really bad habit with heroin and your baby could come out fine. You could be on a really low dose of dihydrocodeine and your baby could have really bad symptoms or the other way about. It just, kind of, totally depends on the baby itself. Everybody's different" Hayley

"Even the ones that are on a lower dose and generally quite stable, who, like you said, it's really unpredictable as to whose baby is going to withdraw" Health Visitor, FG\#3

However, despite wide acknowledgement of the 'unpredictability' of NAS symptoms in relation to opioid use (Cleary et al., 2010), professionals and parents nonetheless focused on reducing drug use (including prescribed drug use) when discussing how to minimise symptoms or incidence of NAS. Here, Hayley responds to a question about advice she would give to other mothers whose babies were at risk of NAS, despite earlier in her interview referring to guidance she had been given by her addictions team about the unpredictable nature of NAS:

"I definitely think there's something in the...if you're on methadone, being on dihydrocodeine is a bit better. [...] So I would maybe say about that, try and get low on that, obviously try and come off it if possible but if not try and go on to dihydrocodeine because it 
Special Issue: Uncertainty

will stay in your system a lot less time. Just try and do everything you can do to make it as least chance possible that the baby's going to... [withdraw]" Hayley

Hayley's account drew on her own experience of transferring from prescribed methadone to dihydrocodeine during pregnancy, and having a baby who was framed as exhibiting only 'mild' symptoms. However, reflecting the 'unpredictability' of NAS, other participants who had not reduced methadone dosage, or who had continued to use illicit drugs during pregnancy, also described babies who were 'fine' and had 'mild' or no symptoms of NAS.

Despite acknowledgement of unpredictability, focusing on maternal drug use as a cause of NAS led to some interviewee and focus group participants providing narratives which alluded to 'blaming' mothers when babies exhibited symptoms of NAS:

"I have to say sometimes when I see... when I've got a baby who is not feeding, not sleeping, and who is all extended and you know ${ }_{L}$ you think to yourself, oh, and it's so hard not to blame the mum, you know, you know yourself, it's trying to rationalise it" Neonatal Nurse, FG\#1

"Interviewer: But [can you talk about] why some of them [babies with NAS symptoms] are really bad and some of them are not so bad.

Liam (father): Just luck nae doubt. It probably depends what the mum's been up to. If the mum's been taking more drugs. No doubt, most people you speak to lie to you about what they've taken. I can guarantee it, nobody will tell you the truth"

Accounts which focused on maternal drug use as a cause of severe symptoms emerged frequently. As in the quote from Liam's interview, this often went alongside a suggestion (posed by parents and professionals) that mothers would not be truthful about their drug use. This narrative coexisted uneasily with acknowledgement of the 'uncertainty' of the relationship between maternal drug dose and severity of NAS.

Uncertainty and diagnosis: scoring babies for NAS Uncertainty regarding NAS extends to the postnatal period, where babies may be monitored for signs of withdrawal. Parents talked of anxieties during pregnancy as to whether their baby might 'have NAS' when it was born. However, in many cases, they spoke of a lack of clarity and inconsistency when discussing the identification or diagnosis of NAS once babies had arrived. This theme emerged especially when discussing the use of 'score charts' to assess babies for presence and severity of symptoms of NAS. In both areas studied, best practice guidance encourages 
Special Issue: Uncertainty

involvement of parents in monitoring babies for signs of NAS, using either an adapted version of Finnegan's Neonatal Abstinence Score Sheet (Area 1) or the Lipsitz Scoring Tool (Area 2)'. However, interviews indicated that in practice parental involvement in scoring was variable. In particular, parents who were involved with the specialist team in Area 1 indicated that they were prepared for the use of a NAS score chart, and encouraged to use it themselves by staff in the team. In contrast, several parents managed by standard maternity services indicated little awareness of the existence of such score charts. Further, parents managed on the postnatal wards suggested scoring was something done by professional staff alone, with no parental involvement. Several parents reported having to ask staff directly about their baby's score, suspecting that had they not asked the chart would not have been mentioned. Joanne was managed by the specialist team and said she had expected to be involved in scoring her baby's NAS symptoms:

"They never even spoke about - like they never even really spoke about the score sheet. I thought it was going to a bigger deal than what it was cause, [specialist midwife], sat and told me all about it one time, Mike [father], was at his group and [the midwife] said that the nurses will go through with me and they'll do it with me in the hospital but nobody ever, nobody ever mentioned her score sheet in the hospital until I mentioned" Joanne

Accounts about parents not being involved in scoring their babies for symptoms of NAS can be understood in a wider context of stigma towards drug using parents, and the threat of losing custody if deemed not to be 'good enough'. Parents' knowledge of the status of their baby in regards to NAS was framed as important, relating to normative concerns with presenting themselves (to staff, the interviewer, themselves) as 'good parents' - interested in their baby's wellbeing. For many parents in our sample this was of special significance, as they had lost custody of older children and were highly sensitised to the risks they faced of losing custody of their youngest child if they were not deemed by statutory services to be 'good enough' parents (Chandler et al., 2013, 2019; Rhodes et al., 2010).

Where babies did appear to have more significant symptoms of NAS, the score chart became a more contentious issue. Shelley and Ryan's baby was described as showing some signs of NAS. As well as being concerned about the symptoms experienced by their baby, the couple spoke of inconsistent messages from staff, and feeling excluded from involvement in the monitoring and care of their baby.

"They didn't even tell me they were doing the test [score chart] half the time. I just knew because the first time they did it, do you know what I mean, and they would fill out the wee 
Special Issue: Uncertainty

thing [score chart] in front of you but they never ever let you see it. I had to ask to see it" Shelley

Across accounts of scoring, parents frequently positioned themselves as relatively powerless knowledge of their baby's NAS status was kept from them by professionals, despite local policies that they be involved in monitoring. At the same time, parents' accounts spoke of attempts to resist this exclusion - asking to see score charts, and presenting themselves as knowledgeable and interested in their baby's care.

The uncertainty of NAS scoring featured throughout these accounts - with frequent tales of disagreements between different staff, and between parents and staff, about the nature and interpretation of a baby's symptoms. These disagreements became particularly acute when considering the potential (threat) of care in the Neonatal Unit (NNU).

Uncertainty and diagnosis: treatment and admission to NNU

Only three participants (including a couple) said their baby was admitted to NNU, and neither of these cases were said to be due to NAS. In other interviews, admission to the NNU was marked as a clear sign that NAS was severe, and that therefore the mother must have been using illicit drugs.

“I've seen with other mums, one girl's dabbling [taking illicit drugs], still kept dabbling compared to me and like, just sticking with methadone, and she's no getting her baby home. [...] her baby's, well, it's in the [neo]natal unit now so it's like, do you know what I mean, your baby's really sick even though you knew the risks" Joanne

Joanne's baby was not treated in NNU, and in the excerpt above she suggests that use of illicit drugs ('dabbling') was a key difference between her and an acquaintance whose baby was treated in NNU and who was also not granted custody ('no getting her baby home'). Again, despite acknowledgement of uncertainty, more 'severe' NAS is associated here with more 'severe' drug use - in this case using illicit opioids in addition to prescribed methadone. Treatment in NNU emerged across the accounts as a clear signifier of 'severe' NAS.

Shelley and Ryan referred to staff members providing inconsistent scores on the NAS chart, and different conclusions as to what form of care would be provided. Both suggested there had been a dispute over whether their baby should be admitted to NNU and treated with opioids in order to relieve symptoms.

"...they would come and tell you, just for instance, "I think, oh she's only scoring a seven" and then we would talk to the midwife later: "oh she's scoring a nine". I says, "well the one 
Special Issue: Uncertainty

told us seven earlier". And then another time they were saying, "oh she's scoring a nine", and then the next thing, "she's scoring 11". But the woman was saying "if it ever goes past a then they go straight to special baby care"" Ryan

Ultimately, Shelley and Ryan's baby was not admitted to NNU, but in both interviews the 'threat' of admission was presented as extremely stressful. Disagreement between parents and medical staff about the diagnosis and treatment of a baby is especially highly charged in the case of NAS - as noted across several interviews, a diagnosis of NAS was understood (and experienced) by some to invoke additional involvement from statutory social work and child protection. Freddie, when reflecting on his concerns about his partners' pregnancy and drug use, noted that "basically, how I saw it if the baby's got NAS you aren't getting your baby". These accounts underline the moral and legal significance of the 'diagnosis' of NAS - with admission to NNU marked as a particularly strong sign a baby had been negatively affected by maternal drug use, which was then connected (by parents and professionals) to parents (mothers') (in)ability to care for their baby at home.

\section{Uncertainty and diagnosis: naming NAS}

Across focus groups with professional staff, contrasting views were raised regarding the importance of 'naming' NAS, and communicating this to parents. This concern sat uneasily with the concurrent narrative that identifying NAS could be challenging, since symptoms may or may not be related to maternal drug use and dose. Uncertainty about naming NAS was reflected in interviews with parents, who tended not to talk about babies as being diagnosed with NAS, but rather of 'some signs' or 'a low score'. Eilidh, for instance, who said her baby was admitted to the neonatal unit for assistance with feeding (not for NAS), reported that her baby:

“...did have slight NAS, but ... not concerning, but enough to make you feel really guilty"

\section{Eilidh}

In Eilidh's account, and among other parents, the presence or absence of NAS, whether and to what extent different symptoms exhibited by babies could be attributed to NAS or something else, often shifted and lacked clarity. It is important to note that these accounts are produced in a context where maternal drug use is highly stigmatised, mothers are framed as morally culpable, and parents face very real legal challenges regarding the custody of their children. Thus, parents' accounts must navigate a complex terrain - acknowledging NAS, but minimising effects to some extent, claiming 'guilt', whilst also trying to live comfortably with their status as (partner of) a 'drug-using mother' who faces blame/shame from multiple sources (Whittaker et al. 2019). 
Special Issue: Uncertainty

In focus groups with professionals, accounts from parents which avoided naming NAS were framed as 'minimising' the diagnosis of NAS. In Focus Group 1, a discussion between a community midwife and neonatal nurse suggested there was a 'problem' with more junior staff failing to clearly communicate a diagnosis of NAS to parents.

Midwife: Yes, and there is, we do get a few woman, you're right, that don't acknowledge and they will try anything else to say, oh it's feeding problems, it's not to do with NAS, and we do try and tell them it is, because it is quite important for them to acknowledge that it is

Neonatal Nurse: But, the reason for that probably is because a lot of the staff who are junior, skirt around the issue with parents.

An interesting contrast can be drawn between the midwife's insistence on the importance of parents 'acknowledging' a baby's symptoms are related to NAS, and the simultaneous narrative that NAS was an uncertain label. The exchange also shows how clinical and social contexts shape uncertainty - some staff may avoid labelling baby's with NAS, perhaps because of their own uncertainty, perhaps because they might see a label of NAS as an unnecessary burden for parents to bear, perhaps because a label of NAS is not necessary in order to provide care for a baby exhibiting symptoms. In contrast, the midwife, who worked in community settings with pregnant and postnatal women who used drugs, argued that naming NAS - and parents acknowledging NAS explicitly - was important. This related to a strong narrative among some professionals that parents should 'take responsibility' for their drug use and the (potential) impacts it might have on their children.

Uncertainty around the diagnosis of NAS was reflected starkly in Joanne and Mike's account of their baby's 'NAS status' changing according to which healthcare professional they were dealing with.

"No, she [Health Visitor] was...she used a score sheet as well. But it went really down, I mean, after the....when the midwife left, we got a new bloody health visitor and she was saying, oh she's stretching. That's NAS. If she's crying a lot, that's NAS. If she's having a burp, that's NAS. We went, what? You're joking" Mike

Mike and Joanne each provided accounts where staff on the postnatal ward had scored their baby increasingly 'low' on the NAS chart, and their community midwife had reassured them that their baby was doing well with no signs of NAS. However, on transfer at 10 days postnatal to the community health visitor, they were told that a wide range of 'symptoms' (none of which had been recorded on the NAS chart they used) could be signs of NAS. For Joanne and Mike - as with other parents - uncertainty was exacerbated by apparent conflicts in the perspective of individual staff regarding whether their baby 'had NAS', the impact of different practices of identification and 
Special Issue: Uncertainty

measurement of symptoms, and the related introduction of a range of apparently disparate symptoms that were attributed to NAS (by the health visitor), but which were not recorded on the NAS chart. It is important to note that in highlighting this case of uncertainty we are not suggesting that a more detailed score chart could have avoided this, made a diagnosis more certain. Rather, this case underlines the instability of NAS, with subtle but important differences between clinical practitioners and contexts of care shaping how NAS is understood and - in Mol's (2002) terms enacted. Such subtle differences nonetheless have far-reaching consequences with regards to NAS, identification of which is associated, as we have demonstrated, with significant maternal/parental guilt and blame (see also Whittaker et al. 2019).

\section{Discussion}

Uncertainty in medicine has long been a topic of sociological inquiry (Atkinson, 1984; Fox, 2000). In this paper we have shown how uncertainty emerges in accounts of parents and professionals when talking about Neonatal Abstinence Syndrome. As has been shown with other conditions, such as Attention-Deficit Hyperactivity Disorder, we found that uncertainty was a feature of talk not just about diagnosis, but also regarding causes and treatments of NAS (Rafalovich, 2005). With NAS the uncertainty that is a feature of conversations about causality, diagnosis, assessment and treatment is particularly fraught due to moral framings and potential legal implications of drug-use in pregnancy (Boyd, 2004). Indeed, our analysis suggests that uncertainty about NAS means that accounts can simultaneously hold the diagnosis and cause of NAS as uncertain, and nevertheless still 'blame mothers' for 'causing' NAS or the possibility of NAS.

NAS can be understood as a social diagnosis, following Brown et al.'s (2011) concept. It is produced by particular political and socioeconomic conditions, though such a reading is rarely foregrounded either in clinical contexts, or in our data. An explicitly political or macro-view of drug use - and outcomes such as NAS - was largely absent in our interviews with parents and professionals, but those conditions nonetheless can be understood as shaping the experience of NAS, or its potentiality, for both health and social care professionals as well as for drug using parents. There was direct evidence of parents' experiences of powerlessness, of feeling marginalised in decisions being made about their own lives and that of their children, in a context where the ultimate threat of losing custody of children was ever-present. These accounts also pointed to the legal and moral frameworks that surround maternal drug use, and which propel the apparent need to diagnose or 'name' NAS, as well as feeding debates about the causes of NAS. We find that despite inherent uncertainty with regard to both the causes and identification or diagnosis of NAS, current 'logics of care' (Whittaker et al. 2019) are enrolled around a focus on individual mothers and their behaviours. 
Special Issue: Uncertainty

Although biomedical framings persist, despite diagnostic uncertainties, these are clearly inflected with notions of moral toxicity.

Brown et al's (2011) explication of social diagnosis does much to shift the focus of diagnosis away from individual bodies, and onto social and political structures which may also be 'diagnosed' as contributing to pathology. In this respect it is useful in allowing a wider reading of the ambiguities and uncertainties that our data and analysis demonstrate are played out in clinical settings. However, this framework has less to offer in terms of considering the intimate, interpersonal contexts in which diagnosis (by multiple social actors) may play out. This issue has been addressed partly in a later paper by Jenkins and Short (2017), who identified the role of resistance to diagnoses as part of any consideration of social diagnosis. However, in each case, practices of diagnosis are left relatively untouched. It is here that Mol's (2002) ethnographic orientation towards practices and meaning-making in relation to the identification of disease by different medical specialities offers additional analytic traction to the concept of social diagnosis. This relates to a need to 'bring bodies back in' as well as considering the social inscriptions within which they are understood.

Paralleling Mol's (2002) arguments about the coexistence of multiple bodies, and multiple enactments of atherosclerosis in a Dutch hospital; our analysis shows that NAS too can be seen as multiple: produced, or enacted, in different ways, through different practices in different places. In the accounts we generated and analysed, NAS was described as being identifiable through different types of score chart, through accumulated professional or lay knowledge and experience. In some cases, NAS was anticipated by parents and some professionals, it was searched for, expected: it retained power as a potentiality even if diagnostic criteria were contested or absent. However, this expectation was not universal: in some clinical settings babies were not monitored or at least, not in front of parents. In other cases, NAS was expected, assumed and assiduously searched for in the form of biomedical evidence. Despite the uncertainty around diagnosis or identification of NAS, in some accounts it appeared that confirmation of NAS was needed: scores might guide treatment pathways - to NNU or not; a confirmed diagnosis of NAS might be seen as simultaneously confirming maternal drug misuse among clinical and lay people alike. Similarly, where NAS was potentially identified, different treatments were possible, with different implications for parents - the NNU in particular, emerged as a site of contestation, and concern. The wider social structures that can so sharply define maternal drug use as harmful and toxic to babies thus play out differently in different contexts. It is this crucial nuancing that Mol's ethnographic approach adds, and which we suggest presents a useful additional perspective to complement the notion of social diagnosis; reminding us to consider carefully the contexts (including power relations) in which diagnosis plays out. 
Special Issue: Uncertainty

Following Fraser et al. (2014), we suggest that uncertainty around NAS may mean there is scope to introduce alternative narratives, and alternative practices of care for babies at risk of 'NAS', that may be experienced as more supportive by parents. This might take the form of resisting scoring of NAS as inherently uncertain and as such a problematic clinical category. Care and treatment could respond instead to the needs of individual babies and their parents, without the explicit naming the 'problem' of NAS. Alternatively, the 'diagnosis' of NAS could be framed more explicitly as a 'social diagnosis' - one which is understood by parents and professionals alike as relating to the complex interplay between biological and sociological factors - including entrenched inequalities and marginalisation facing many parents who use drugs. This approach might serve to shift attention away from the role of 'maternal drug use' in producing NAS, towards a broader range of social challenges faced by parents. Perhaps in drawing on the concept of social diagnosis we might connect negative, stigmatising framings of drug use in pregnancy to the intersection of socioeconomic, gender and (in some contexts) racial inequalities, with those most affected by punitive responses to drug use in pregnancy often being those with the least power. Approaching NAS as a social diagnosis, while recognising its multiple framings, may enable responses to clinical uncertainty which avoid, rather than promote, further marginalisation of parents who use drugs.

Such suggestions pose significant challenges, though, in terms of how NAS might be otherwise (Mol, 1999). Indeed, we have shown how currently uncertainties around NAS provide space for some of the more punitive responses reported in our data and elsewhere (Boyd 1999, Knight 2015), rather than the more caring alternatives that were occasionally reported in our data. Our analysis also provokes significant political questions about who might choose from a range of different productions of NAS and how easily alternative (less stigmatising) approaches to responding to drugexposed infants might be incorporated into current practice, given prevailing (negative) cultural attitudes towards addiction and motherhood.

One way in which such questions might be at least partly resolved is by promoting a more inclusive culture in the clinical setting in which diagnoses are made. For instance, the SENSES framework (Nolan et al., 2003) focuses on inclusive decision making between service providers and service users (including family). Of importance is the creation of an operational culture in which staff and service users share a philosophy of care which values people rather than tasks (perhaps including diagnosis, or 'proving' the presence or absence of NAS). This may provide a framework which is able to empower service providers and families to challenge existing practice and decision making in a space where seeks to recognise all parties as experts, and where existing tensions between each may exist. This approach has been used with promising initial indications in family-focused approaches in adult 
Special Issue: Uncertainty

drug services (Orr et al., 2014), and as such may provide a useful approach in reimagining care for families affected by NAS.

\section{Acknowledgements}

This project was funded by Edinburgh and Lothian Health Foundation (ELHF), grant reference 10314. The paper benefitted greatly from feedback from anonymous reviewers and editors. Special thanks to all of the parents and professionals who took part; as well as those who supported recruitment for the study.

\section{References}

ACMD (2003). Hidden Harm: responding to the needs of children of problem drug users. London: Home Office.

Atkinson, P. (1984). Training for certainty. Social Science \& Medicine, 19, 949-956.

Atwood, E.C., Sollender, G., Hsu, E., Arsnow, C., Flanagan, V., Celenza, J., et al. (2016). A Qualitative Study of Family Experience With Hospitalization for Neonatal Abstinence Syndrome. Hospital Pediatrics, 6, 626-632.

Benoit, C., Stengel, C., Marcellus, L., Hallgrimsdottir, H., Anderson, J., MacKinnon, K., et al. (2014). Providers' constructions of pregnant and early parenting women who use substances. Sociology of Health \& IIIness, 36, 252-263.

Blaxter, M. (1978). Diagnosis as category and process: The case of alcoholism. Social Science \& Medicine. Part A: Medical Psychology \& Medical Sociology, 12, 9-17.

Boyd, S.C. (1999). Mothers and Illicit Drugs: Transcending the Myths. Toronto: University of Toronto Press.

Boyd, S.C. (2004). From witches to crack moms: women, drug law, and policy. Durham: Carolina Academic Press.

Brown, P. (1990). The name game: Toward a sociology of diagnosis. Journal of Mind and Behavior, 11, 385-406.

Brown, P., Lyson, M., \& Jenkins, T. (2011). From diagnosis to social diagnosis. Social Science \& Medicine, 73, 939-943.

Chandler, A., Whittaker, A., Cunningham-Burley, S., Williams, N., McGorm, K., \& Mathews, G. (2013). Substance, structure and stigma: Parents in the UK accounting for opioid substitution therapy during the antenatal and postnatal periods. International Journal of Drug Policy, 24, e35-e42.

Cleary, B.J., Donnelly, J., Strawbridge, J., Gallagher, P.J., Fahey, T., Clarke, M., et al. (2010). Methadone dose and neonatal abstinence syndrome-systematic review and meta-analysis. Addiction, 105, 2071-2084.

Cleveland, L.M., \& Bonugli, R. (2014). Experiences of Mothers of Infants with Neonatal Abstinence Syndrome in the Neonatal Intensive Care Unit. Journal of Obstetric, Gynecologic \& Neonatal Nursing, 43, 318-329.

Flavin, J., \& Paltrow, L.M. (2010). Punishing Pregnant Drug-Using Women: Defying Law, Medicine, and Common Sense. Journal of Addictive Diseases, 29, 231-244.

Fox, R.C. (2000). Medical Uncertainty Revisited. In G.L. Albrecht, R. Fitzpatrick, \& S.C. Scrimshaw (Eds.), Handbook of Social Studies in Health and Medicine. London: SAGE Publications Ltd.

Fraser, S., Moore, D., \& Keane, H. (2014). Habits: Remaking Addiction. Basingstoke Palgrave Macmillan.

Fraser, S., \& valentine, k. (2008). Substance and Substitution. Basingstoke: Palgrave Macmillan. Gregory, J. (2010). (M)Others in Altered States: Prenatal Drug-Use, Risk, Choice, and Responsible Self-Governance. Social \& Legal Studies, 19, 49-66. 
Hennink, M., Hutter, I., \& Bailey, A. (2011). Qualitative Research Methods. London: Sage.

Hudak, M.L., Tan, R.C., Frattarelli, D.A.C., Galinkin, J.L., Green, T.P., Neville, K.A., et al. (2012). Neonatal Drug Withdrawal. Pediatrics, 129, e540-e560.

Jansson, L.M., \& Velez, M. (2012). Neonatal abstinence syndrome. Current Opinion in Pediatrics, 24, 252-258 210.1097/MOP.1090b1013e32834fdc32833a.

Jenkins, T.M., \& Short, S.E. (2017). Negotiating intersex: A case for revising the theory of social diagnosis. Social Science \& Medicine, 175, 91-98.

Jutel, A. (2009). Sociology of diagnosis: a preliminary review. Sociology of Health \& IIIness, 31, 278299.

Jutel, A., \& Nettleton, S. (2011). Towards a sociology of diagnosis: Reflections and opportunities. Social Science \& Medicine, 73, 793-800.

Kenny, K.S., Barrington, C., \& Green, S.L. (2015). "I felt for a long time like everything beautiful in me had been taken out": Women's suffering, remembering, and survival following the loss of child custody. International Journal of Drug Policy, 26, 1158-1166.

Kilty, J.M., \& Dej, E. (2012). Anchoring Amongst the Waves: Discursive Constructions of Motherhood and Addiction. Qualitative Sociology Review, 9, 6-23.

Klee, H., Jackson, M., \& Lewis, S. (2002). Drug misuse and motherhood. London: Routledge.

Knight, K.R. (2015). addicted. pregnant. poor. Durham: Duke University Press.

Knopf, A. (2016). Baby's mother is the best treatment for neonatal abstinence syndrome. Alcoholism \& Drug Abuse Weekly, 28, 1-4.

Marcellus, L. (2007). Neonatal abstinence syndrome: reconstructing the evidence. Neonatal Network: NN, 26, 33-40.

McQueen, K., Murphy-Oikonen, J., \& Desaulniers, L. (2015). Maternal Substance Use and Neonatal Abstinence Syndrome: A Descriptive Study. Maternal and Child Health Journal, 19, 17561765.

Mol, A. (1999). Ontological politics. A word and some questions. The Sociological Review, 47, 74-89.

Mol, A. (2002). The Body Multiple: Ontology in Medical Practice. Durham: Duke University Press.

Morris, M., Seibold, C., \& Webber, R. (2012). Drugs and having babies: An exploration of how a specialist clinic meets the needs of chemically dependent pregnant women. Midwifery, 28, 163-172.

Nolan, M., Grant, G., Keady, J., \& Lundh, U. (2003). New directions for partnerships:Relationshipcentred care. In M. Nolan, U. Lundh, G. Grant, \& J. Keady (Eds.), Partnerships in family care: Understanding the care giving career. Maidenhead: Open University Press.

Orr, L.C., Elliott, L., \& Barbour, R.S. (2014). Promoting family-focused approaches within adult drug services: The potential of the 'Senses Framework'. International Journal of Drug Policy, 25, 888-896.

Powis, B., Gossop, M., Bury, C., Payne, K., \& Griffiths, P. (2000). Drug-using mothers: social, psychological and substance use problems of women opiate users with children. Drug and Alcohol Review, 19, 171-180.

Pryor, J.R., Maalouf, F.I., Krans, E.E., Schumacher, R.E., Cooper, W.O., \& Patrick, S.W. (2017). The opioid epidemic and neonatal abstinence syndrome in the USA: a review of the continuum of care. Archives of Disease in Childhood - Fetal and Neonatal Edition, 102, F183.

QSR International Pty Ltd. (2012). NVivo qualitative data analysis software; Version 10.

Radcliffe, P. (2009). Drug use and motherhood: strategies for managing identity. Drugs and Alcohol Today, 9, 17-21.

Radcliffe, P., Flávia Pires Lucas d'Oliveira, A., Lea, S., dos Santos Figueiredo, W., \& Gilchrist, G. (2016). Accounting for intimate partner violence perpetration. A cross-cultural comparison of English and Brazilian male substance users' explanations. Drug and Alcohol Review, n/a-n/a.

Rafalovich, A. (2005). Exploring clinician uncertainty in the diagnosis and treatment of attention deficit hyperactivity disorder. Sociology of Health \& IIIness, 27, 305-323. 
Rhodes, T., Bernays, S., \& Houmoller, K. (2010). Parents who use drugs: Accounting for damage and its limitation. Social Science \& Medicine, 71, 1489-1497.

Riessman, C.K. (2008). Narrative Methods for the Human Sciences. Thousand Oaks: Sage.

Shriver, T.E., \& Bodenhamer, A. (2018). The enduring legacy of black lung: environmental health and contested illness in Appalachia. Sociology of Health \& IIIness, 40, 1361-1375.

Sword, W., Jack, S., Niccols, A., Milligan, K., Henderson, J., \& Thabane, L. (2009). Integrated programs for women with substance use issues and their children: a qualitative meta-synthesis of processes and outcomes. Harm Reduction Journal, 6.

Vrecko, S. (2010). Birth of a brain disease: science, the state and addiction neuropolitics. History of the Human Sciences, 23, 52-67.

Whittaker, A., Chandler, A., Cunningham-Burley, S., Midgley, P., Elliott, L., \& Cooper, S. (2019). Problematisation and regulation: Bodies, risk, and recovery within the context of Neonatal Abstinence Syndrome. International Journal of Drug Policy, 68, 139-146.

Wolff, K., \& Perez-Montejano, R. (2014). Opioid neonatal abstinence syndrome: controversies and implications for practice. Curr Drug Abuse Rev, 7, 44-58.

\footnotetext{
' In each of these cases the charts indicate higher scores for more severe symptoms.
} 